\title{
PLM customizing: Results of a Qualitative Study with Industrial Experts
}

\author{
Ezgi Sucuoglu ${ }^{1}$, Konrad Exner ${ }^{2}$, Rainer Stark ${ }^{1,2}$ \\ ${ }^{1}$ Technische Universität Berlin, Berlin, Germany \\ ezgi.sucuogluecampus.tu-berlin.de \\ rainer.stark@tu-berlin. de \\ ${ }^{2}$ Fraunhofer Institute for Production Systems and Design Technology, Berlin, Germany \\ konrad.exnereipk. fraunhofer. de \\ rainer.starkipk.fraunhofer.de
}

\begin{abstract}
The implementation and utilization of a product lifecycle management (PLM) system, including the continuously adoption to business processes, methods and functions, implies massive challenges and outlay for organizations. Despite the importance of customizing in PLM projects, there are no adequate models to support organizations in their customizing process. This paper focuses on the customizing process of PLM systems considering not only the technical IT view, but also the organizational and the human context. In order to identify the state of the art in industrial practice eleven qualitative interviews have been conducted. The results and implications are presented in this paper. The findings comprise five dimensions and an additional generic PLM customizing process.
\end{abstract}

Keywords: PLM Customizing, Customizing Process, PLM in Industry

\section{Introduction}

The company specific adaption of PLM software is one of the major challenges during the PLM implementation process. Furthermore, configuration and customization is the main cost driver regarding PLM implementation, see Figure 1.These initial investments will even be increased due to the need of continuous maintenance and support. Aggravating this effect is guaranteeing the release compatibly of the adapted PLM solution. However, PLM customizing is often indispensable due to a broad variety of constraints and demands. Firstly, from an IT system perspective companyspecific information standards and security measures have to be observed and are often managed by a configuration of the PLM system. Secondly, the PLM IT solution needs to be optimally integrated in the overall IT deployment, thus interfaces needs to be considered and potentially configured or developed. Thirdly, from an organizational and user-centered perspective the PLM solution has to meet certain requirements regarding business processes and functionality. For instance, internal and external collaboration and communication is increasing and has to be optimally supported with PLM systems [1]. Therefore, the modification of the standard - out of the box 
(OOTB) or commercially over the shelf (COTS) - PLM solution is only avoidable with great limitations for these three considerations. Additionally, new frameworks which integrate the different perspectives such as organization, IT, data models, engineering activities etc. have to be utilized in order to conduct systematic change processes [2]. Eventually, the efficiency and effectivity of PLM customizing in reference to, for instance, business process can hardly be measured in practice.

Challenges

Costs

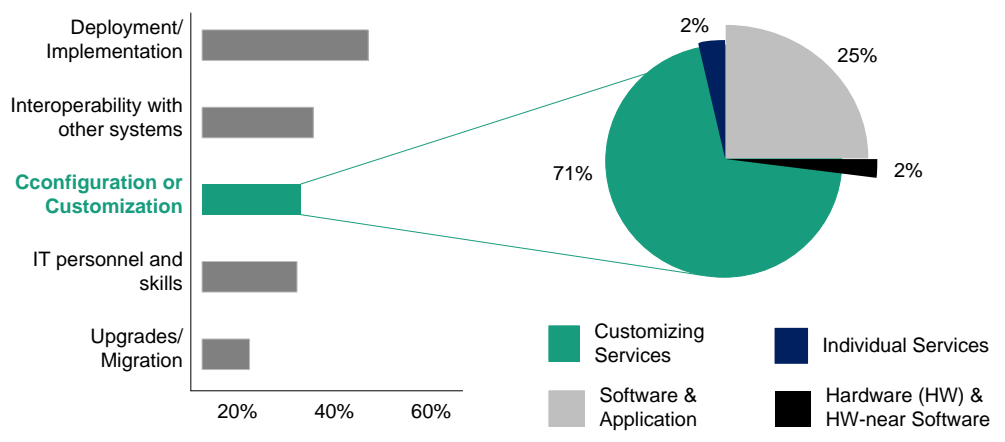

Fig. 1. Impact of Customizing in PLM implementation (based on [3], [4])

In theory, product lifecycle management including strategies, software implementation and organizational background has been addressed intensively [5, 6, 7]. Customizing and configuration is addressed as an option to optimally suit PLM software to the customers' needs. Nevertheless, a discussion of differences between customizing and configuration as well as the advantages and disadvantages is rarely conducted. A rare exclusion is the quite extensive distinction between tailoring and customizing by [8]. Nevertheless, further approaches or guidelines regarding the adaption of an existing PLM system is missing. Therefore, in spite of this great affect, PLM implementation lacks certain PLM customizing specific methods and approaches which address these challenges. For this reason, many companies developed their own practices, which are often based on expert knowledge. In order to identify commonalities and differences and integrate them in a state of the art from an industrial point of view a qualitative study has been planned and conducted. The two main research questions are:

1. How is PLM customizing defined and conducted in industrial practice?

2. How can the added value of PLM customizing be measured?

The research approach including an extensive description of the procedure regarding the qualitative study is described in chapter 2 . The results of the case are presented in chapter 3 and comprise a demographic overview of the participants as well as the findings of the study. In chapter 4 the overall results will be discussed. 


\section{Research Approach}

The research approach is based on the Design Research Methodology (DRM) [9] and covers a comprehensive research plan. The scope of this contribution covers step 1 and 2, marked green in Figure 2.

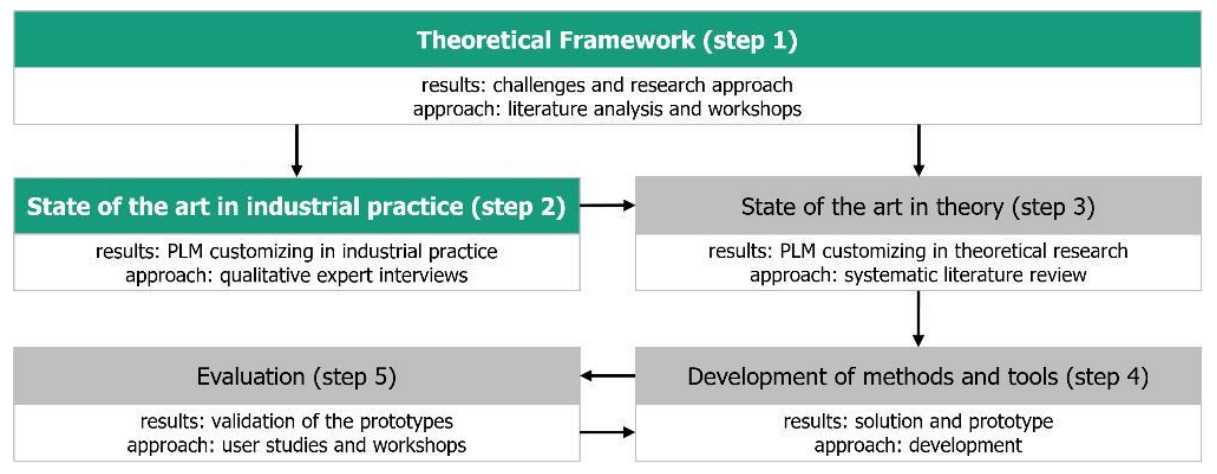

Fig. 2. Research approach

Based on an initial literature analysis and workshops including discussions with industrial and research experts a lack of comprehensive description of PLM customizing has been identified. Therefore, the state of the art in industrial practice (step 2) as well as in theoretical research (step 3) need be assed. For this reason, a qualitative study has been planned and conducted.

\subsection{Study Design}

The study focuses three analyzing dimension with three clusters of questions each, which are given in table 1 .

Table 1. Study Focus und Questions

\begin{tabular}{|l|l|}
\hline Dimension & Questions \\
\hline & $\begin{array}{l}\text { - How is the overall PLM strategy defined and communicated } \\
\text { within the company? }\end{array}$ \\
$\begin{array}{l}\text { Organization } \\
\text { and processes }\end{array}$ & $\begin{array}{l}\text { How is PLM customizing defined, which phases are standard- } \\
\text { ized and for what reason is PLM customizing conducted? } \\
\text { - Which stakeholders with dedicated responsibilities are partici- } \\
\text { pating in the PLM customizing process and which criteria are } \\
\text { defined for each phase? }\end{array}$ \\
\hline $\begin{array}{l}\text { - Which IT-systems are used for PLM? } \\
\text { IT systems and }\end{array}$ & $\begin{array}{l}\text { How are IT-standards and guidelines as well as collaboration } \\
\text { defined across different divisions and locations? }\end{array}$ \\
& $\begin{array}{l}\text { - How is the performance and efficiency of PLM customizing } \\
\text { measured? }\end{array}$ \\
\hline
\end{tabular}




\begin{tabular}{|l|l|}
\hline Dimension & Questions \\
\hline $\begin{array}{l}\text { Human, activi- } \\
\text { ties and meth- } \\
\text { ods }\end{array}$ & $\begin{array}{l}\text { - How many users does the PLM system have and how is their } \\
\text { usage intensity and purpose differentiated? } \\
\text { they use specific and formalized methods? }\end{array}$ \\
\hline $\begin{array}{l}\text { How are the user and their specific requirements integrated in } \\
\text { the PLM customizing process? }\end{array}$ \\
\hline
\end{tabular}

Based on this clarification a specific interview type has been chosen: episodic interviews. A particular feature is the change between storytelling request in order to capture episodically knowledge and open questions in order to derive semantic knowledge $[10,11]$. This procedure provides sufficient means to derive formalized and informal knowledge from the interviewers. In accordance to the method and the questions of the analyzing dimension, a questionnaire has been developed and pretested with a senior PLM consultant. The purpose of the pre-test is verification of the questions regarding [12]:

- interest and attention of the interviewed experts,

- continuity of interview procedure,

- effect of the interview structure and duration of the interview.

The results of the pre-test required a change in the procedure and the clarification of some questions. Overall, the interview has been rated as very interesting and of high relevance.

The target groups are medium-sized and large companies with at least 250 employers in order to ensure a certain amount of PLM users. The interview participants shall address different views on PLM customizing. According to these constraints eleven interview partners from seven companies participated in this study. A demographic overview and the results are discussed in chapter 3

\section{$3 \quad$ Findings}

The findings of the study are aggregated in three main sections. In the first section the demographic data of the study including information to the experts and their organization is given. The sustentative evaluation is analyzed separately in the second section to enable anonymity for participants. The last section represents the generic process of PLM customizing.

\subsection{Demographic Evaluation}

The demographic evaluation obtains information to organization (industry and number of employees), PLM IT systems (PLM software and number of users) and participants (PLM experience in years, position and education). Figure 3 illustrates the demographic data of the study. 


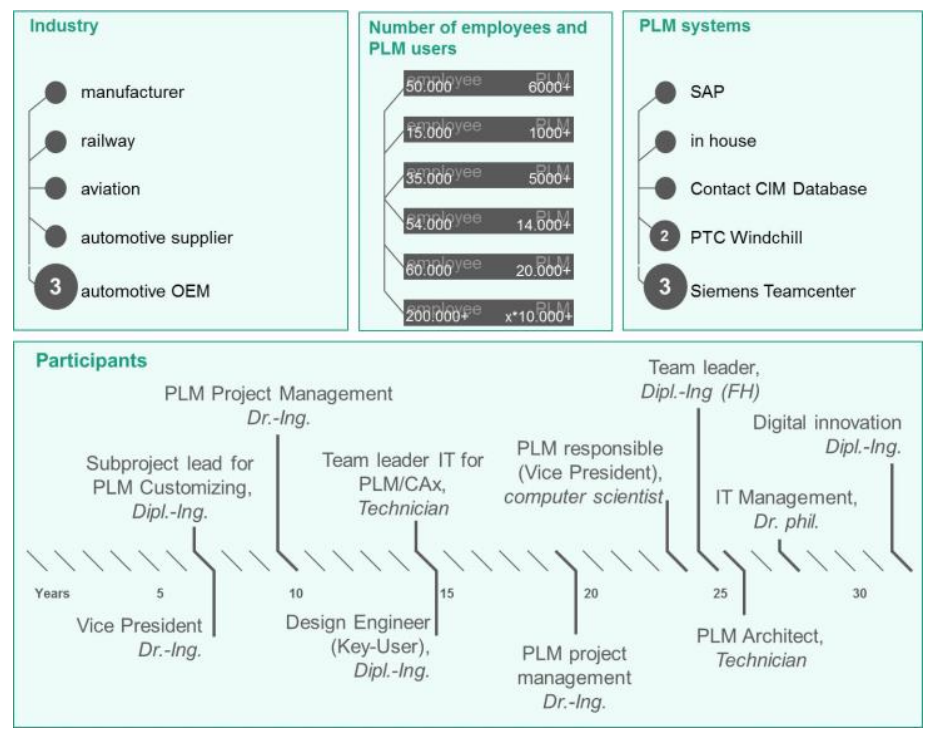

Fig. 3. Demographic Data ${ }^{1}$

One important aspect has been in acquiring participants of different branches and different size, which could be successfully achieved by integrating five different branches with companies ranging from 15.000 employees to over 200.000. Additionally, the interviewees represented experts with many years of experience in PLM.

\subsection{Substantive Evaluation}

In this paper the substantive evaluation focuses on five clusters which are derived from the three dimensions and belonging questions of the study given in Table 1.

Scope and understanding of customizing. Customizing can include not only configuration but also a complex system expansion. It is important to choose the adequate level of change considering the specific context. The initial step is to analyze the understanding of PLM customizing. Therefore three perspectives are considered:

- Scope of Customizing describes to what extent the systems is adapted

- Prioritization of the scope describes the preferred adoption variant

- Strategic Focus demonstrates two views for performing PLM customizing

Figure 4. summarizes the answers of the experts in these perspectives and clarifies the bride scope of different interpretations for customizing of PLM systems.

1 The numbers dedicate the amount of nominations; no number refers to one nomination. 


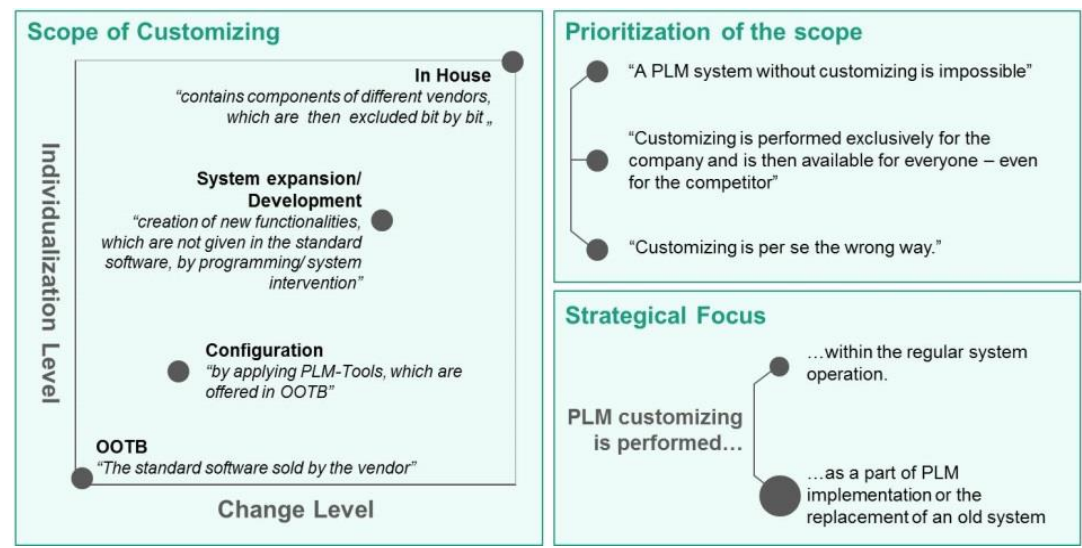

Fig. 4. Scope and understanding of PLM customizing

PLM Customizing comprises every deviation of an OOTB solution considering the level of individualization and the level of change. In house solutions, which are in our case a bundle of adopted systems from different vendors ${ }^{2}$, have the highest level of individualization and change, since they are developed for and adopted to the special needs of the organization. There is no general way to perform PLM customizing. An in house solution can be the best way for one organization and the maximum use of OOTB functionalities for the other.

Motivation for PLM customizing. The drivers to render customizing in PLM projects are analyzed regarding to three aspects. The role of management describes on which organizational level the necessity of PLM customizing is initiated. With the decision for performing the customizing the focus of proceeding the PLM customizing should also been set, which can be either the lead of the methods or the lead of the business processes. Besides this high level approach, specific causes are determined to execute the customizing of the PLM system. Figure 5 presents the state of the art for the motivation of PLM customizing according to the study considering the three aspects described above.

2 Organization which develops or acquires software for selling. 


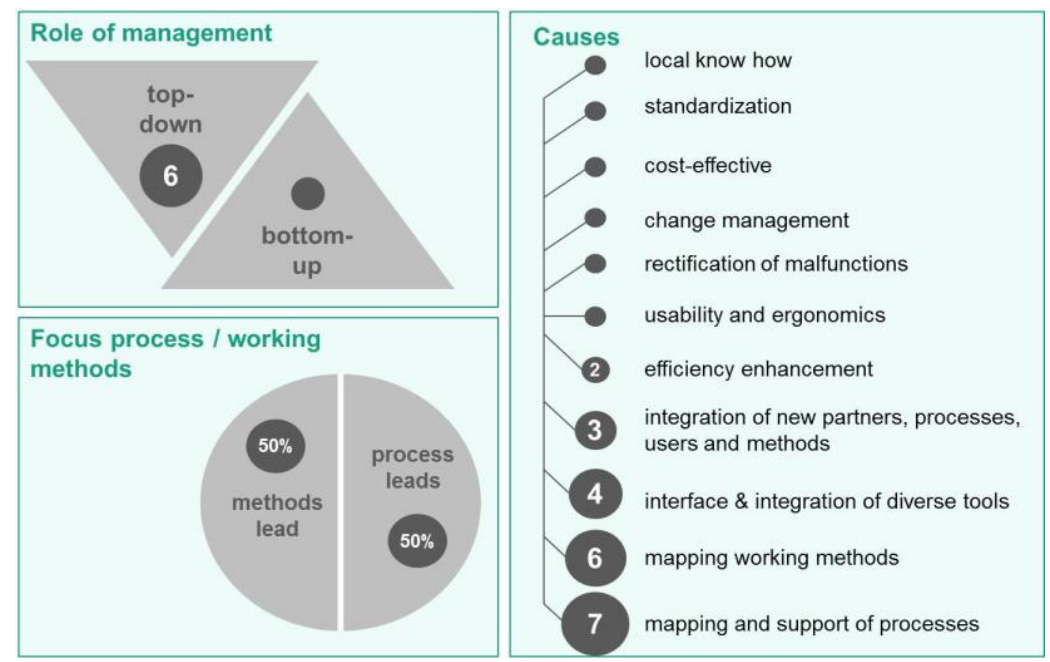

Fig. 5. Motivation for PLM customizing

The initiation is mostly top-down. Whereas the focus lies on both the methods and the business processes. The main reasons for customizing PLM are to adapt the working methods and the lived process to the software.

Challenges of PLM customizing. The study analyzes PLM customizing by not only contemplating the IT and system view but also the organizational und user-centered view. Therefore the human context represents the biggest challenge for customizing PLM systems (see Figure 6).

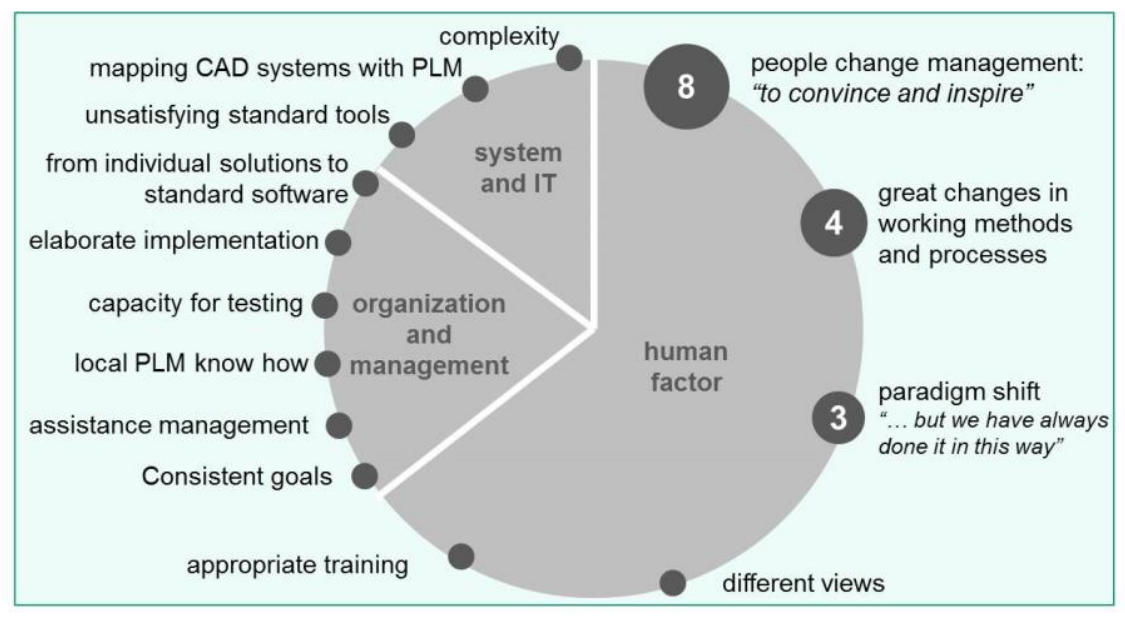

Fig. 6. Challenges of PLM customizing 
Especially people change management and the inspiration and conviction of the users for a change constitutes a significant issue for organizations.

Collaboration model. The collaboration with external service providers and the vendor is a common practice for customizing PLM systems. However the collaboration model differs from strategic to operative level.

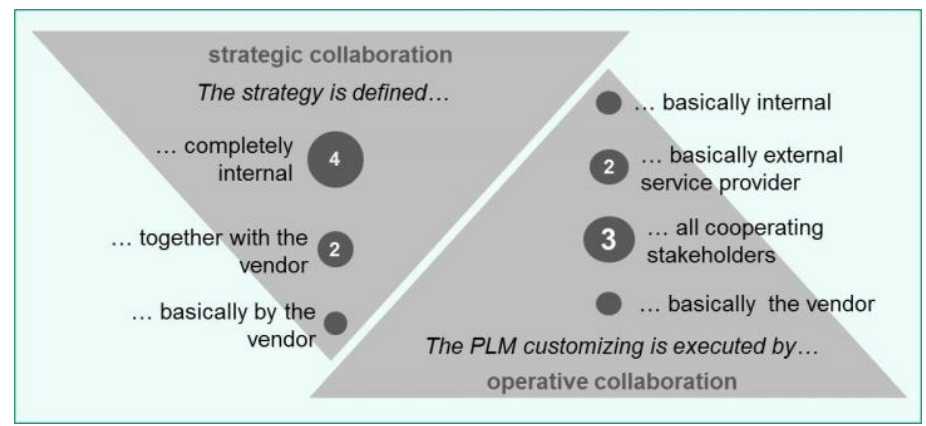

Fig. 7. Collaboration models for PLM customizing

Organizations mostly prefer to define their PLM customizing strategy internally, whereas, the operative execution is usually defined as an activity for external providers or cooperating stakeholders (see Figure 7).

Impact and traceability. Based on the results of the study only few or none models to measure the efficiency of PLM customizing by retracing could be identified.

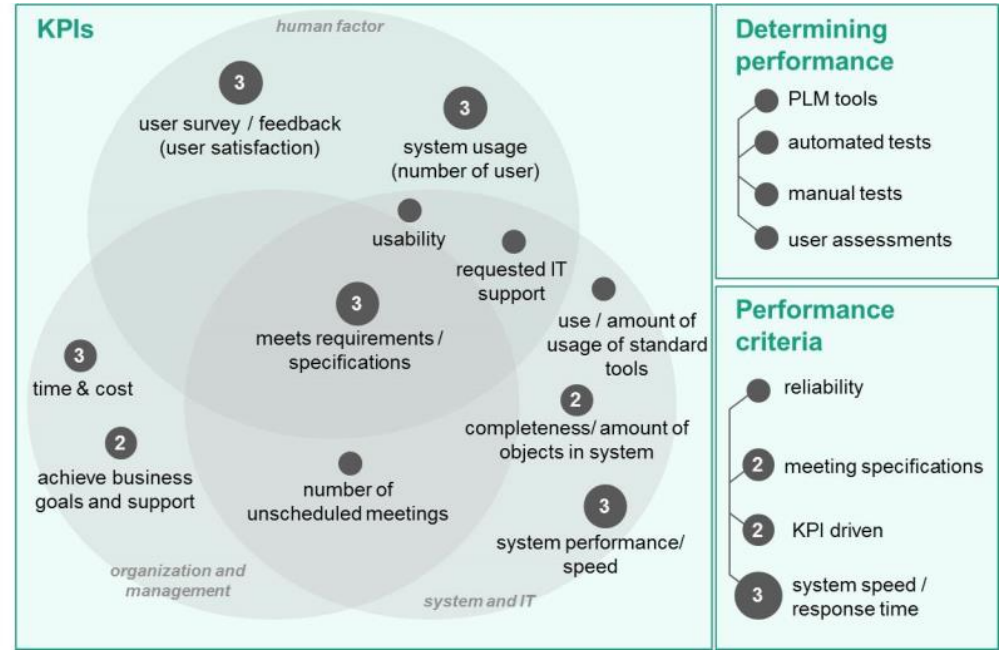

Fig. 8. The impact and traceability of PLM and customizing 
It is hardly possible to make a statement about the impact of a customized part in a PLM system environment. Experts agreed on the necessity of such indicators and models which allow organization to measure and also ensure the success of PLM customizing. Nonetheless, organizations define KPIs and performance criteria to determine the performance of the PLM system and its customizing with different tools (see figure 8).

\subsection{The Process of PLM customizing}

The generic process comprises all steps mentioned by the experts to perform the customizing of PLM systems. Customizing starts with taking up the requirements of the users in form of a specific definition or formulated wishes. Subsequently, the requirements are analyzed and evaluated considering the confirmation to the strategy, processes and methods. In a next step the requirements have to be clustered, analyzed and prioritized in order to release them in the steering group. For the operative implementation the documentation and the voting with partners has to be conducted. Following the specification, consultation and planning the final release has to be given by the steering board. The development and programming can either be done internally or externally despite to the collaboration model selected (see Figure 7). The testing with key users, PLM team and IT can contain acceptance and usability test, consistency of process tests, system performance test, live-tests on system and tests with user cases on a test instance. The approval of customizing and the related documentation is usually done by the key user and the management of the specialist department. After implementing the customized part in the PLM environment and the official roll-out, the process ends with final documentation and adoption of methods and trainings. The generic process is represented in Figure 9.

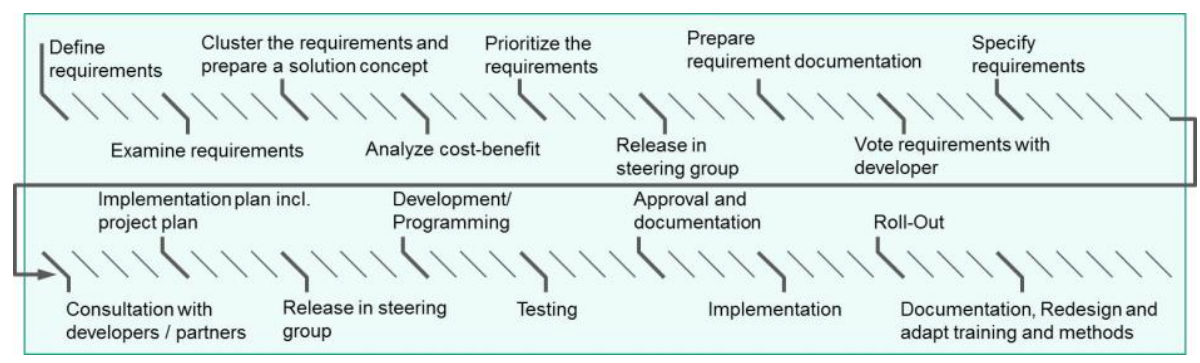

Fig. 9. The generic process of PLM customizing

Experts mentioned that besides this defined process small changes and customizing parts also are done separately with a small group of expert to shorten the time. Depending to the priority and urgency this work around can also be applied by bigger changes. 


\section{Conclusion and Outlook}

This contribution comprises the extensive research results of qualitative study regarding PLM customizing. The results indicate a broad variety of strategies and perspectives addressing the challenges of adopting PLM systems to organizational needs. Besides specific findings discussed within chapter 3, three general statements could be derived. First of all is PLM customizing interpreted as a term differently, also within the same organization. Basically, only one company could certainly provide a specific differentiation between configuration, customization, modification etc. Nevertheless, in order to understand short-term and long-term costs and benefits a sound definition is strongly recommended. Secondly, PLM customizing should not only be addressed as an issue of IT. A user-centered approach including experts for PLM methods is a key success factor in order to overcome diverse challenges. Thirdly, the success of PLM customizing is almost exclusively measured by fulfilling the users' requirements and standard project KPIs. Nevertheless, the long term changes in affectivity and efficiency of the conducted changes are not analyzed at all. Considering the vast cost of PLM customizing, finally, tracking the customized modules and measuring its success is a great opportunity for companies.

In a next step a comprehensive and systematic literature review of PLM customizing will be conducted in order to complete these finding with the research perspective on PLM customizing. Eventually, a method will be developed which includes metrics to conduct and evaluate PLM customizing along the complete process, from the initial assessment to the long term tracking of the results.

\section{Acknowledgments}

The authors would like to express their sincere gratitude to the participants of the study. Their insights and expert knowledge based on years of experience provided invaluable information to this topic.

\section{References}

[1] Lünnemann, P., Müller, P., Hayka, H., Wang, M., Kirsch, L., Neumeyer, S., 2016. Zukunft der unternehmensübergreifenden Kollaboration.: Expertenmeinungen zu aktuellen Herausforderungen und zukunftsweisenden Trends in der kollaborativen Produktentwicklung, 1. ed 1. Band., Berlin.

[2] Damerau, T., Hayka, H., Neumeyer, S., Woll, R., Stark, R., 2014. Intelligent Information Technologies to Enable Next Generation PLM, in: Product Lifecycle Management for a Global Market. Revised Selected Papers. 11th IFIP WG 5.1 International Conference, PLM2014, Yokohama, Japan. 7-9 Juli 2014. Springer, Heidelberg, Berlin, pp. 485-495.

[3] Jackson, C., 2010. The CIO's Role in PLM: Facilitation "Great Product" Development to Drive Economic Recovery. 
[4] Eigner, M., 2014. Out of the Box oder doch Customizing?: Product Lifecycle Management (PLM). Produktdaten Journal 21 (2), 46-49.

[5] Eigner, M., Stelzer, R., 2009. Product Lifecycle Management: Ein Leitfaden für Product Development und Life Cycle Management, 2., neu bearbeitete Aufl. ed. Springer, Dordrecht [u.a.].

[6] Sendler, U., 2009. Das PLM-Kompendium: Referenzbuch des ProduktLebenszyklus-Managements. Springer, Berlin [u.a.].

[7] Feldhusen, J., Gebhardt, B., 2008. Product Lifecycle Management für die Praxis: Ein Leitfaden zur modularen Einführung, Umsetzung und Anwendung. Springer, Berlin [u.a.], 1 online resource (Online-Ressource.).

[8] Best, O., 2015. PLM: Configuration v Customization. Let's sort it out... http://beyondplm.com/2015/10/06/plm-configuration-v-customization-lets-sort-itout/. Accessed 22 February 2017.

[9] Blessing, L.T.M., Chakrabarti, A., 2009. DRM, a design research methodology. Springer, Dordrecht, New York, 1 online resource (xvii, 397.

[10]Flick, U., 2011. Das Episodische Interview, in: Oelerich, G., Otto, H.-U. (Eds.), Empirische Forschung und Soziale Arbeit. Ein Studienbuch. VS Verlag für Sozialwissenschaften / Springer Fachmedien Wiesbaden, Wiesbaden, Wiesbaden, pp. 273-280.

[11]Kaiser, R., 2014. Qualitative Experteninterviews: Konzeptionelle Grundlagen und praktische Durchführung. Springer VS, Wiesbaden, Online-Ressource (XIII, 157 S. 17 Abb, online resource).

[12]Schnell, R., Hill, P.B., Esser, E., 2011. Methoden der empirischen Sozialforschung, 9., aktualis. Auflage ed. Oldenbourg, R, München, 640 S. ; 